Maciej Gnass' ${ }^{1}$ Anna Filarecka ${ }^{2}$, Artur Bartczak ${ }^{3}$, Małgorzata Szołkowska ${ }^{4}$, Agnieszka Knapczyk ${ }^{3}$, Monika Lis ${ }^{3}$, Monika Skrobot ${ }^{3}$, Jerzy Soja ${ }^{5}$, Magadlena Misiaszek ${ }^{6}$, tukasz Barszczok Damian Czyżewski ${ }^{7}$, Artur Szlubowski ${ }^{1,6}$

'Endoscopy Unit, Pulmonary Hospital, Zakopane, Poland

${ }^{2}$ Department of Pulmonology, Pulmonary Hospital, Zakopane, Poland

${ }^{3}$ Department of Pathology, Pulmonary Hospital, Zakopane, Poland

${ }^{4}$ Department of Pathology, Institute of Tuberculosis and Lung Diseases, Warsaw, Poland

${ }^{5}$ Department of Medicine, Jagiellonian University, Cracow, Poland

${ }^{6}$ Endoscopy Unit, John Paul II Hospital, Cracow, Poland

${ }^{7}$ Chair and Department of Thoracic Surgery of Silesian Medical University, Katowice, Poland

\title{
Transbronchial lung cryobiopsy guided by radial mini-probe endobronchial ultrasound in interstitial lung diseases - a multicenter prospective study
}

Abstract

Introduction: Transbronchial lung cryobiopsy (TBLC) is commonly used in diagnosing interstitial lung diseases (ILDs). A general anesthesia with endotracheal intubation, balloon blockers and fluoroscopy control is the most common modality. Simplifying the procedure without decreasing it's safety could result in wider use.

Prospective, observational study was conducted in three Polish pulmonology centers to evaluate safety and diagnostic yield of TBLC under conscious sedation, without intubation and bronchial blockers and with radial-EBUS guidance instead of fluoroscopy. Material and methods: In patients suspected of ILD, in accordance with high resolution computer tomography (HRCT) selected lung segments were examined with radial-EBUS mini probe without a guide sheath. If the lung infiltrations were visible this locations were preferred. If not, specimens were taken from two different segments of the same lobe. Two to five biopsies with freezing time 5-8 seconds were performed. Moreover ultrasound examination was used to avoid injury of lung vessels.

Results: From March 2017 to September 2019 - 114 patients (M: 59, F: 55) of mean (SD) age 54 (14) years were included to the study on the basis of medical history and HRCT. Histopathology was conclusive in 90 (79\%) patients and included 16 different diagnoses (sarcoidosis, EAA, COP predominantly). 24 inconclusive biopsies of unclassifiable pulmonary fibrosis were followed up. Complications included five cases $(4.4 \%)$ of pneumothorax requiring a chest tube drainage and a minor and moderate bleeding in few cases. There was no need for use of balloon bronchial blockers.

Conclusions: TBLC under conscious sedation guided by radial EBUS mini-probe is novel, reasonable and safe technique for histological diagnosis of ILDs.

Key words: cryobiopsy, interstital lung diseases, endoscopy, endobronchial ultrasound, bronchoscopy

Adv Respir Med. 2020; 88: 123-128

\section{Introduction}

Interstitial lung disease (ILD), also known as diffuse parenchymal lung disease is a heterogeneous group of conditions. The common pathological mechanism of these illnesses is the presence of inflammation and/or fibrosis in the lung parenchyma. When clinical outcome and radiology findings (especially in high resolution computed tomography, HRCT) are not sufficient to establish proper diagnosis, a pathological assessment of the involved lung tissue may be necessary in selected

Address for correspondence: Maciej Gnass, Endoscopy Unit, Pulmonary Hospital, Zakopane, Poland; e-mail: m.gnass@gmail.com

DOI: 10.5603/ARM.2020.0086

Received: 17.11.2019

Copyright (C) 2020 PTChP

ISSN 2451-4934 
patients. A transbronchial lung biopsy (TBLB) with endoscopic forceps is the most common and the least invasive approach. Whereas its high value in diagnosing only few ILDs (as sarcoidosis) is well established, TBLB is not recommended for identifying pulmonary fibrosis. Regarding idiopathic pulmonary fibrosis (IPF), either ATS/ /ERS/JRS/ALAT (American Thoracic Society/European Respiratory Society/Japanese Respiratory Society/Latin American Thoracic Association) or Fleischner Society guidelines recognize a surgical lung biopsy (SLB, usually video-assisted thoracic surgery lung biopsy - VATS-LB) as a gold standard and recommend it if HRCT is not conclusive $[1,2]$. The other important indication for lung biopsy (LB) in patients with diffuse lung infiltrations is an exclusion of malignancy. Considering that the most common primary lung cancer - adenocarcinoma is quite often diagnosed among patients with no smoking history and with radiological appearance of diffuse lung infiltrates imitating ILD, histological assessment is crucial in many cases $[3,4]$. Patients after VATS-LB often require a few days of hospitalization and face a risk of some possible complications, as prolonged air leak and bronchopleural fistula, involving in some cases reoperation and low but significant mortality risk $[5,6]$. Considering all these complications, the development and widespread introduction of minimally invasive methods of LB into the practice in ILD with pulmonary fibrosis is necessary.

A biopsy with the use of a flexible cryoprobe was introduced in clinical practice a few years ago, and initially proved its diagnostic value both in endobronchial and transbronchial tissue sampling [7, 8]. Studies published to date have suggested that diagnostic yield of transbronchial lung cryobiopsy (TBLC) in establishing proper diagnosis of ILD reaches 70-80\% [9-12].

The review of cryobiopsy literature has revealed a lack of TBLC procedure standardization $[1,10,11]$. While the basic technique of TBLC is nearly the same, the approach how to avoid and manage the most common complications: pneumothorax and severe bleeding differs. The risk of pneumothorax increases when a cryoprobe is inserted too far into the bronchi and freezing hurts the visceral pleura. On the other hand, when a cryoprobe is too close to the pulmonary vessels, the risk of severe bleeding is higher.

There is no doubt that choosing a correct site for $\mathrm{LB}$ and controlling the cryoprobe position during the procedure is crucial both for its efficacy and safety. The most common method of supervising a position of a cryoprobe's tip is fluoroscopy [10-12].
In our center, a radial endobronchial ultrasound miniature probe (r-EBUS) is routinely used for diagnosing peripheral solid and semisolid lung lesions. A few years ago we introduced r-EBUS before performing conventional forceps TBLB in diagnosing patients suspected of ILD. In our experience in some cases, if lung opacities are visible in ultrasound imaging, r-EBUS allows the bronchoscopist to choose the best biopsy site. It is very useful in localizing vessels of the hilum and sometimes even shows the visceral pleura. Based on these observations, we designed a study for the diagnosis of ILDs with the use of TBLC without fluoroscopy but controlled with r-EBUS.

\section{Material and methods}

This study was designed as an open-label observational prospective trial. It was a multicenter study conducted in Pulmonary Hospital, Zakopane, in John Paul II Hospital, Cracow, and in Clinical Hospital of the Silesian Medical University, Katowice, Poland. A study protocol was approved by Bioethical Committee of District Medical Association, Cracow, Poland (129/KBL/ /OIL/2017).

Consecutive patients in whom the decision of lung biopsy was made, in the process of ILD diagnosing were included in the study. Qualification for LB was made on the basis of clinical outcome and radiology assessed by experts in pulmonology and radiology according to the British Thoracic Society and previous edition of ATS/ERS/JRS/ALAT guidelines [13, 14]. The main exclusion criteria were as follows: lack of patient's agreement, age below 18 years and standard contraindications for invasive endoscopy such as inappropriate hemostasis parameters, instable cardiovascular disease and/or heart failure and pulmonary hypertension. After receiving the informed consent, the TBLC procedure was performed. During the endoscopy, the patient was in the supine position under conscious sedation: fentanyl (0.05-0.1 mg) and midazolam $(2.5-7.5 \mathrm{mg})$ were administered intravenously. A pulse oximetry monitoring was mandatory and oxygen supply was provided when it was necessary. A whole procedure was performed by a trained endoscopist with the assistance of two endoscopy nurses. A flexible videobronchoscope (BFT180 or BFT190 Olympus, Tokyo, Japan) with a 2.8-3.0 mm working channel was introduced orally, without endotracheal intubation with a tube or rigid bronchoscope. After topical administration of a $2 \%$ lignocaine and bronchial 


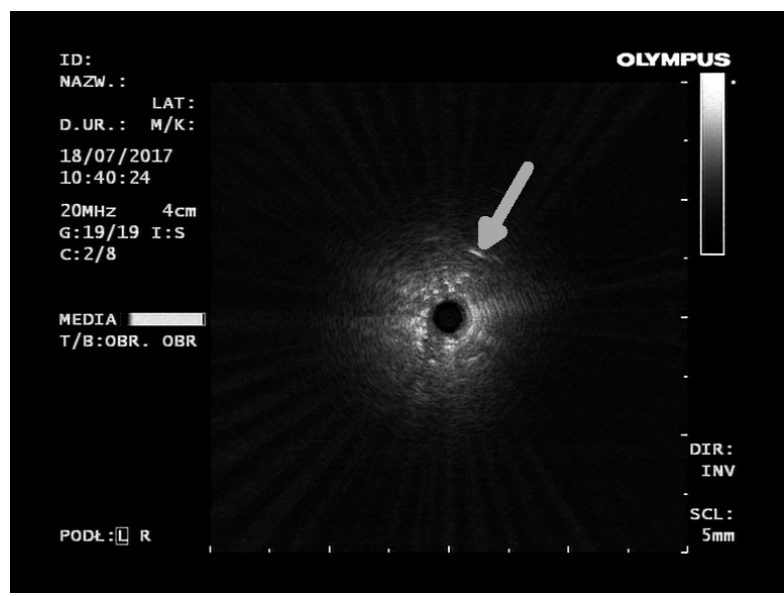

Figure 1. Ultrasound imaging of the visceral pleura (white arrow) obtained with radial endobronchial ultrasound mini-probe — rEBUS. With the consent of the Archives of Endoscopy Unit of Pulmonary Hospital, Zakopane

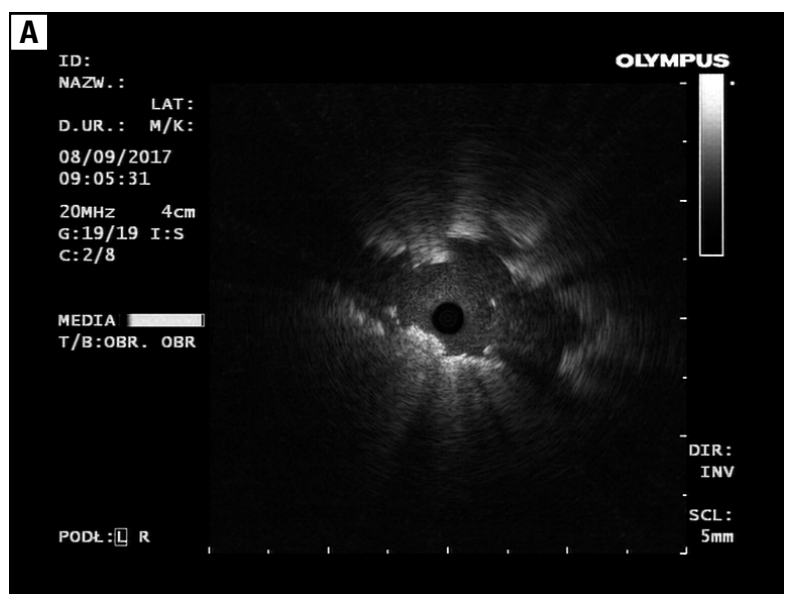

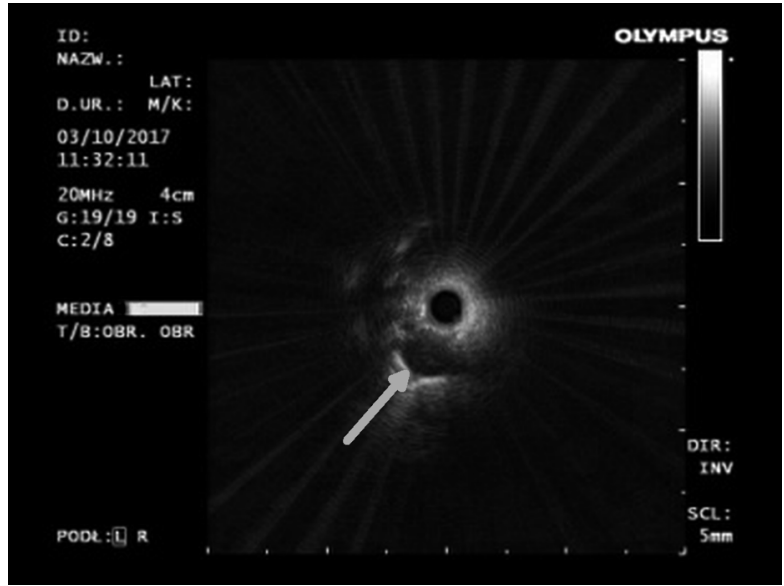

Figure 2. Ultrasound imaging of the subsegmental pulmonary artery (white arrow) obtained with radial endobronchial ultrasound mini-probe (rEBUS). Biopsy in this site would cause significant bleeding. With the consent of the Archives of Endoscopy Unit of Pulmonary Hospital, Zakopane

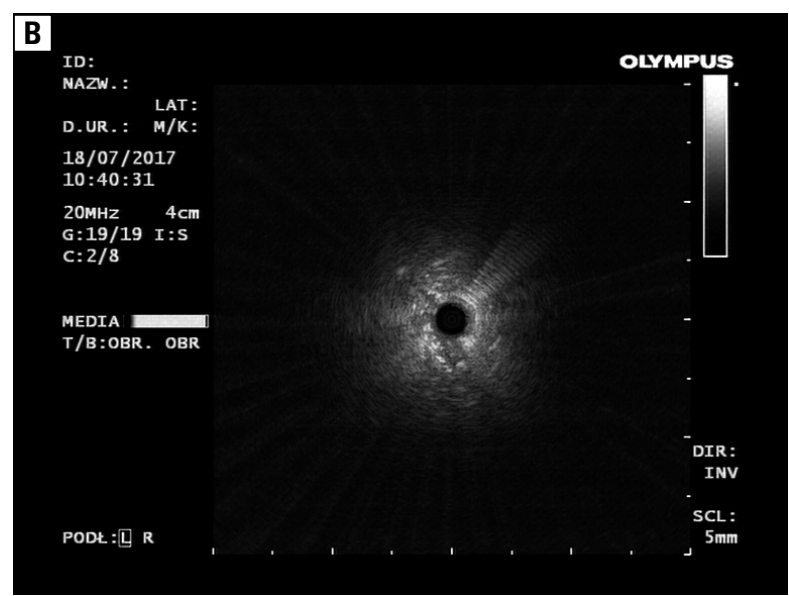

Figure 3. Ultrasound imaging of interstitial lung infiltration obtained with radial endobronchial ultrasound mini-probe (rEBUS). A. Cryptogenic organizing pneumonia; B. Usual interstitial pneumonia. With the consent of the Archives of Endoscopy Unit of Pulmonary Hospital, Zakopane

tree inspection, the ultrasound radial miniature probe UM-S20-20R (Olympus, Tokyo, Japan) was used. Selected, according to HRCT, lung regions were examined with r-EBUS in order to choose the optimal site for TBLC. The ultrasound probe without a guide sheath was inserted as far as possible (sometimes a specific ultrasound sign of the visceral pleura was visible - Figure 1). A slow withdrawing of the ultrasound probe with controlling the distance between the pleura (if visible) and hilar vessels (Figure 2) allowed to identify the safest area for the biopsy. The additional advantage of this method was that in some cases the lung infiltrates were visible in the ultrasound image (Figure 3), and if there were no vessels close to the lesions, the exact location of the biopsy could be established. Following an ultrasonographic examination, the flexible cryoprobe (diameter $1.9 \mathrm{~mm}$, length $900 \mathrm{~mm}$; ERBE, Tubingen, Germany) was introduced to the working channel of the bronchoscope. After placing its tip in previously selected site (according to r-EBUS examination), TBLC was performed with freezing time of 5-8 sec (mean $7 \mathrm{sec}$ ). Two to five biopsies were taken from two different segments of the same lobe, whereas in diffused lung infiltrates, lower lobes were chosen. If ultrasound examination was negative for the presence of lung infiltrations, distance from the visceral pleura was approximately 1-2 $\mathrm{cm}$. The bronchoscope with the inserted cryoprobe and lung tissue adhered to the tip of the probe was taken out of the airways en bloc. As the specimen was placed in the saline (Figure 4), the cryoprobe was pulled out, and the same scope was inserted into the bronchial tree 


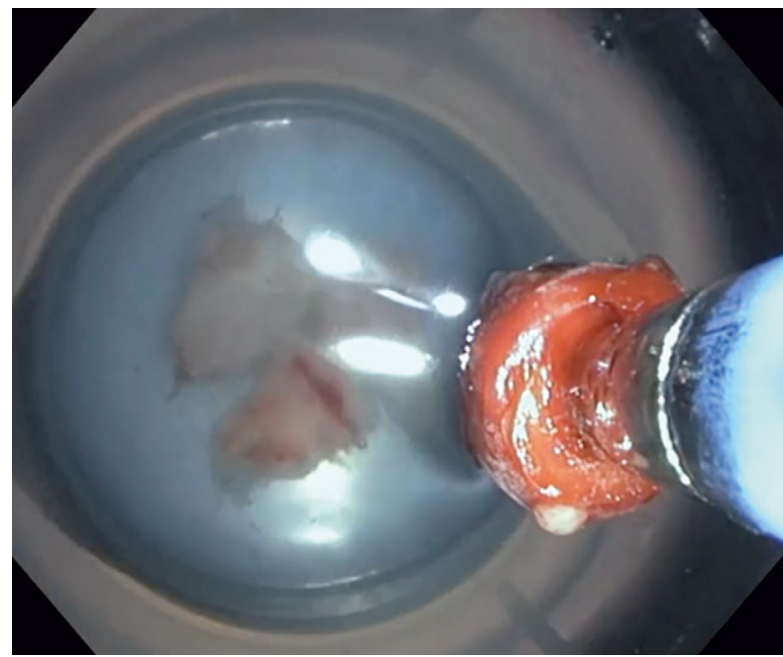

Figure 4. Two lung tissue specimens obtained with flexible cryoprobe and the third one attached to the cryoprobe's tip. With the consent of the Archives of Endoscopy Unit of Pulmonary Hospital, Zakopane

again to assess and, if necessary, to manage the bleeding. The time without eye control of the bronchial tree was about 15-30 seconds. The balloon catheter for lobar occlusion after TBLC was not used. For safety reasons, the double-lumen endotracheal tube was prepared in case of major bleeding requiring patient's intubation and separated lung ventilation. The bleeding after biopsy was qualified as minor, moderate and major according to its management: minor - no action needed, self-limiting bleeding; moderate - bleeding managed endoscopically (cold saline, adrenaline solution, segmental bronchi blockage with the scope); major when additional actions were needed (intubation, admission to the intensive care, surgery). The chest X-ray due to the pneumothorax control was done within 2-4 hours after TBLC or immediately according to the physician decision. After the procedure, the specimens were transferred from saline to a $10 \%$ formaldehyde solution and then sent to the Pathology Department, where were assessed by two independent pathologists after standard Hematoxylin \& Eosin (HE) staining (Figure 5). The final diagnosis was established in the discussion by the multidisciplinary team comprising experts in pulmonology, radiology and lung pathology.

\section{Statistical analysis}

A statistical analysis was conducted on the basis of descriptive statistic as the arithmetic mean with standard deviation. The results were used to calculate the diagnostic yield.

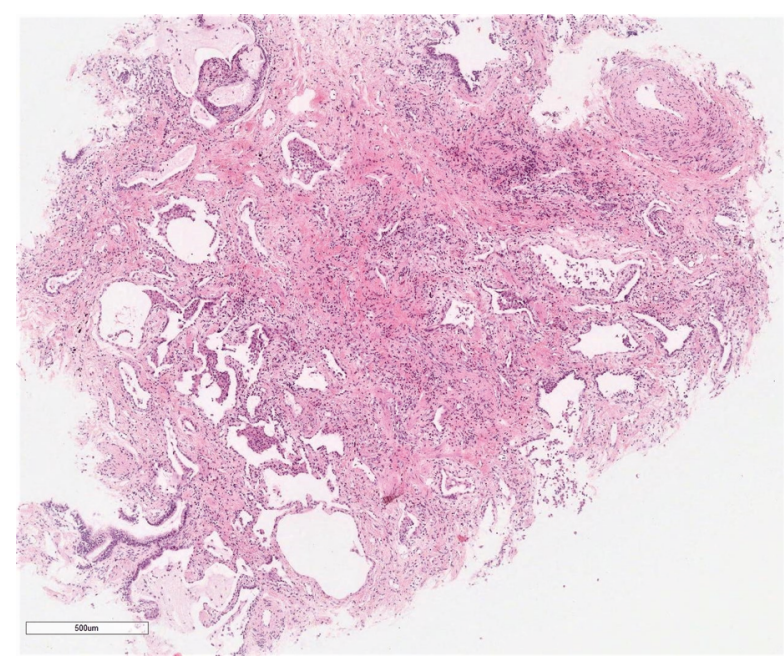

Figure 5. A microscopic imaging of cryobiopsy (hematoxilin and eosin staining). Usual interstitial pneumonia (UIP) associated with histologic pattern (patchy fibrosis and honeycomb). With the consent of the Archives of Pathology Department of Pulmonary Hospital, Zakopane

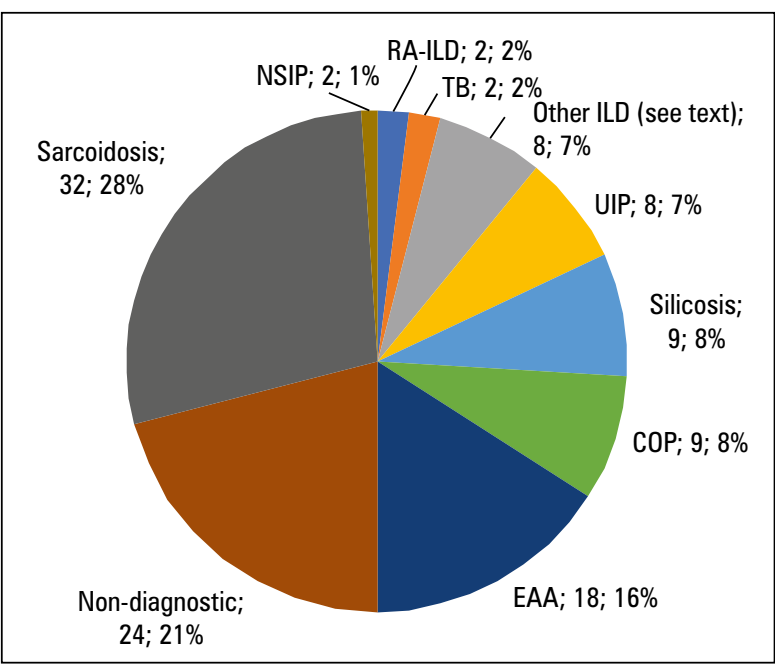

Figure 6. Histological diagnoses in a group of 114 patients with ILD $(\mathrm{n} ; \%)$. EAA - extrinsic allergic alveolitis; COP — cryptogenic organizing pneumonia; ILD — interstitial lung disease; NSIP — nonspecific interstitial pneumonia; RA-ILD — rheumatoid arthritis-related ILD; TB — tuberculosis; UIP — usual interstitial pneumonia.

\section{Results}

From March 2017 to September 2019 one hundred and fourteen patients, 59 (52\%) males and 55 (48\%) females of mean (SD) age 54 (14) years were enrolled in the study. The time of the whole procedure was 10-20 min (mean $15 \mathrm{~min}$ ). The lung tissue obtained by r-EBUS-TBLC was found sufficient for pathological evaluation in all cases. A diameter of specimen was 5-10 mm (mean $7 \mathrm{~mm}$ ). Histopathology was conclusive for final diagnosis in 90 (79\%) cases. The most com- 
mon histological diagnosis was sarcoidosis -32 (28.1\%) patients, then extrinsic allergic alveolitis (EAA) - $18(15.8 \%)$ cases, silicosis and cryptogenic organizing pneumonia (COP) $-9(7.9 \%)$ individuals each. Usual interstitial pneumonia (UIP) was diagnosed in 8 (7\%) cases. Tuberculosis, rheumatoid arthritis-related ILD (RA-ILD) and non-specific interstitial pneumonia (NSIP) were diagnosed in two cases each, and hemosiderosis, Langerhans cell histiocytosis (LCH), lymphocytic interstitial pneumonia (LIP), exogenous lipoid pneumonia (ELP), smoking-related interstitial fibrosis (SRIF), vasculitis, scleroderma and mixed connective tissue disease-related ILD (SCL-ILD and MCTD-ILD) in one case each (Figure 6). Among twenty four non-diagnostic biopsies, unclassifiable lung fibrosis and normal lung (in few cases with the presence of macrophages with brown deposits characteristic for smokers) were the most common pathological findings. Patients with non-conclusive TBLC are followed up and further subgroup analyses are planned after establishing final diagnoses for the whole group.

Complications included only five cases $(4.4 \%)$ of pneumothorax requiring a chest tube drainage and minor and moderate bleeding in few cases.

\section{Discussion}

Whereas specimens obtained by conventional TBLB are often found by pathologists not adequate for histological evaluation because they are too small and damaged by forceps, those obtained by TBLC are bigger and free of crushing artifacts. In some studies, the results of TBLC in ILD were even comparable to VATS-LB, and the procedure was safer [9]. A diagnostic yield of TBLC in different reports varies from 51\% to $98 \%$. Among the procedure-related complications, the most common is pneumothorax $(0-33 \%)$. Other reported complications include serious bleeding up to $20 \%$, acute exacerbation (AE) of ILD up to $4 \%$ and death in most cases due to AE-IPF (up to $4 \%$ ) [10-12]. The results of our study have shown the diagnostic yield on the level of $79 \%$ and the complication rate of $4.4 \%$ (exclusively pneumothorax). No serious bleeding was noted in our study. It can be connected with guidance method we used to visualize the pulmonary vessels. Almost all data about the safety of TBLC come from studies with fluoroscopic guidance. In two studies, in which authors didn't use any guidance for TBLC, complication ratio was $0 \%$ and $12 \%$ for serious bleeding and $4.7 \%$ and $8 \%$ for pneumothorax [15, 16].
Taking into consideration the mentioned data and our results in safety area, it is disputable whether the use of fluoroscopy has significant impact on the procedure safety. More data, especially from comparative randomized trials is needed.

The high percentage of sarcoidosis diagnosed in our study (28.1\%) must be noted and commented due to its possible influence on the diagnostic yield. It must be underlined that only patients without lymphadenopathy (stage III and IV) and with negative conventional bronchoscopy (endobronchial mucous biopsy, TBLB) were included in the study.

Although the usefulness of TBLC for collecting adequate samples seems to be out of the question, there are no standards when it comes to the exact procedure technique. The fluoroscopic guidance, endotracheal tube or rigid bronchoscope intubation with deep sedation or general anesthesia is the most common way of performing TBLC. For safety reasons, it is important to know if a freezing tip of the flexible cryoprobe is distant enough both from the chest wall and hilar vessels. In twenty six of TBLC reports from 2009 to 2017 analyzed by Lentz RJ et al., only in 2 studies fluoroscopic guidance was not used (in one only in $40 \%$ cases) [10].

Experts preparing recommendations for IPF management very carefully analyzed data regarding safety and efficacy of the TBLC, and they found it promising but not standardized enough to be widely recommended. They have pointed out that "a standardized procedure for lung cryobiopsy that optimizes the balance between diagnostic yield and complications needs to be developed among experts currently engaged with the procedure" [1]. They have suggested the experts already performing this procedure should work on its standardization and do not recommend introducing this procedure by new centers before its value and safety will be determined. However it seems to be a very cautious statement, but it might be justified in the light of recently published data showing very poor concordance between TBLC and surgical lung biopsy (SLB). Romagnoli et al. showed that TBLC would have led to a different treatment if SLB was not performed in 11 of 21 (52\%) cases [17]. This small but well-designed study cooled down the enthusiasm about TBLC in ILDs and showed a lack of strong data comparing SLB with TBLC.

Our idea of using r-EBUS control simplifies the whole procedure and avoids X-ray exposure without decreasing diagnostic yield, and the safety of the procedure is high. Whereas the 
fluoroscopy provides guidance mainly for safety reasons, the r-EBUS assessment of the lung before TBLC may also sometimes help to choose the most proper biopsy site (because some types of infiltrations are visible in ultrasound image). Although cost/effectiveness analysis was not the purpose of the study, in our opinion, it is worth noticing that simplifying the method (no use of endotracheal intubation, balloon blockers or fluoroscopic control) should lead to the cost reduction.

At the time of preparing our study protocol, there were no data regarding its efficacy and safety profile. During last years a few papers were published and summarized by Gupta et al. [18]. Most of the analyzed studies presented the role of r-EBUS in peripheral nodule cryobiopsy. The authors found and examined only three studies (including our preliminary report in 20 patients) describing the role of r-EBUS cryobiopsies in diffuse parenchymal lung disease. In their conclusions they have found it to be a feasible and safe procedure with high diagnostic yield. The studies on the role of r-EBUS-guided TBLC are currently under way (i.e. in Vanderbilt University Medical Center). In our opinion, this technique should be further investigated and compared in randomized trials, both with TBLC under fluoroscopic control and balloon bronchial blockers in intubated patients (how most centers perform it) and with SLB. Without comparative studies, strong recommendation on the use and method of performing TBLC in ILDs will be impossible.

\section{Conclusions}

The results of our study have shown that combination of r-EBUS and TBLC is a novel, reasonable and safe technique to diagnose ILDs. It could be an alternative way of performing TBLC in ILD patients but further comparative trials are required.

\section{Conflict of interest}

None declared.

\section{References:}

1. Raghu G, Remy-Jardin M, Myers JL, et al. An official ATS/ERS/ JRS/ALAT statement: idiopathic pulmonary fibrosis: evidence-based guidelines for diagnosis and management. Am J Respir Crit Care Med. 2011; 183(6): 788-824, doi: 10.1164/rccm.2009040GL, indexed in Pubmed: 21471066.

2. Lynch DA, Sverzellati N, Travis WD, et al. Diagnostic criteria for idiopathic pulmonary fibrosis: a Fleischner Society White Paper. Lancet Respir Med. 2018; 6(2): 138-153, doi: 10.1016/ S2213-2600(17)30433-2, indexed in Pubmed: 29154106.
3. Imaizumi K. Clinical features of primary lung cancer presenting as pulmonary consolidation Mimicking pneumonia. Fujita Med J. 2016; 2: 17-21.

4. Ismail M, Sekhon R. Diffuse pulmonary infiltrates: A guise of adenocarcinoma. Respir Med Case Rep. 2017; 22: 150153, doi: 10.1016/j.rmcr.2017.08.003, indexed in Pubmed: 28831374.

5. Krasna MJ, White CS, Aisner SC, et al. The role of thoracoscopy in the diagnosis of interstitial lung disease. Ann Thorac Surg. 1995; 59(2): 348-351, doi: 10.1016/0003-4975(94)00844-w, indexed in Pubmed: 7847948.

6. Utz JP, Ryu JH, Douglas WW, et al. High short-term mortality following lung biopsy for usual interstitial pneumonia. Eur Respir J. 2001; 17(2): 175-179, doi: 10.1183/09031936.01.17201750, indexed in Pubmed: 11334116.

7. Babiak A, Hetzel J, Krishna G, et al. Transbronchial cryobiopsy: a new tool for lung biopsies. Respiration. 2009; 78(2): 203-208, doi: 10.1159/000203987, indexed in Pubmed: 19246874.

8. Franke KJ, Theegarten D, Hann von Weyhern C, et al. Prospective controlled animal study on biopsy sampling with new flexible cryoprobes versus forceps: evaluation of biopsy size, histological quality and bleeding risk. Respiration. 2010; 80(2): 127-132, doi: 10.1159/000287251, indexed in Pubmed: 20160432.

9. Ravaglia C, Bonifazi M, Wells AU, et al. Safety and diagnostic yield of transbronchial lung cryobiopsy in diffuse parenchymal lung diseases: a comparative study versus video-assisted thoracoscopic lung biopsy and a systematic review of the literature. Respiration. 2016; 91(3): 215-227, doi: 10.1159/000444089, indexed in Pubmed: 26926876.

10. Lentz RJ, Argento AC, Colby TV, et al. Transbronchial cryobiopsy for diffuse parenchymal lung disease: a state-of-the-art review of procedural techniques, current evidence, and future challenges. J Thorac Dis. 2017; 9(7): 2186-2203, doi: 10.21037/ jtd.2017.06.96, indexed in Pubmed: 28840020.

11. Hetzel J, Maldonado F, Ravaglia C, et al. Transbronchial cryobiopsies for the diagnosis of diffuse parenchymal lung diseases: expert statement from the cryobiopsy working group on safety and utility and a call for standardization of the procedure. Respiration. 2018; 95(3): 188-200, doi: 10.1159/000484055, indexed in Pubmed: 29316560.

12. Ravaglia C, Wells AU, Tomassetti S, et al. Diagnostic yield and risk/benefit analysis of trans-bronchial lung cryobiopsy in diffuse parenchymal lung diseases: a large cohort of 699 patients. BMC Pulm Med. 2019; 19(1): 16, doi: 10.1186/s12890-019-0780-3, indexed in Pubmed: 30651103.

13. Bradley B, Branley HM, Egan JJ, et al. Interstitial lung disease guideline: the British Thoracic Society in collaboration with the Thoracic Society of Australia and New Zealand and the Irish Thoracic Society. Thorax. 2008; 63 Suppl 5: v1-58, doi: 10.1136/ thx.2008.101691, indexed in Pubmed: 18757459.

14. Raghu G, Collard HR, Egan JJ, et al. An official ATS/ERS/JRS/ ALAT statement: idiopathic pulmonary fibrosis: evidence-based guidelines for diagnosis and management. Am J Respir Crit Care Med. 2011; 183(6): 788-824, doi: 10.1164/rccm.2009-040GL, indexed in Pubmed: 21471066.

15. DiBardino DM, Haas AR, Lanfranco AR, et al. High complication rate after introduction of transbronchial cryobiopsy into clinical practice at an academic medical center. Ann Am Thorac Soc. 2017; 14(6): 851-857, doi: 10.1513/AnnalsATS.201610-829OC, indexed in Pubmed: 28231021.

16. Bango-Álvarez A, Ariza-Prota M, Torres-Rivas H, et al. Transbronchial cryobiopsy in interstitial lung disease: experience in 106 cases - how to do it. ERJ Open Res. 2017; 3(1), doi 10.1183/23120541.00148-2016, indexed in Pubmed: 28344982.

17. Romagnoli M, Colby TV, Berthet JP, et al. Poor concordance between sequential transbronchial lung cryobiopsy and surgical lung biopsy in the diagnosis of diffuse interstitial lung diseases. Am J Respir Crit Care Med. 2019; 199(10): 1249-1256, doi: 10.1164/rccm.201810-1947OC, indexed in Pubmed: 30864813.

18. Gupta A, Youness H, Dhillon SS, et al. The value of using radial endobronchial ultrasound to guide transbronchial lung cryobiopsy. J Thorac Dis. 2019; 11(1): 329-334, doi: 10.21037/ jtd.2018.10.116, indexed in Pubmed: 30863611. 